\title{
Tópicos y focos iniciales en el español hablado: funciones pragmáticas y correlatos formales
}

\section{Las tres dimensiones de la estructura de la información}

En este capítulo se parte de un modelo de la estructura de la información que se compone de tres subdimensiones distintas, y que parece haber alcanzado durante los últimos años un grado de aceptación relativamente alto. ${ }^{1}$ La primera dimensión del modelo concierne al grado de familiaridad asumida de los referentes discursivos en la cognición de los interlocutores en el momento de la realización del enunciado (cf. Prince 1981). A nivel discursivo los referentes codificados en una oración ${ }^{2}$ se pueden clasificar como dados o nuevos en el discurso (cf. Prince 1981, 225ss.), o como (no) inferibles/accesibles por el cotexto lingüístico, o sea a partir de constituyentes explícitamente dados (bridging), o por el contexto extralingüístico, es decir por la situación y/o el saber (general o específico) por parte de los locutores (cf. Prince 1992, 304ss.).

La segunda dimensión se refiere a la posibilidad del hablante de estructurar los elementos a nivel oracional. Este puede empezar la oración con un constituyente que representa la base de predicación para los elementos que siguen, y optar así por una articulación tópico-comentario (TC): “[. . .] the speaker announces a topic and then says something about it” (Hockett 1958, 201). De acuerdo con Hockett, el criterio decisivo que se adopta en este capítulo para la definición del término tópico es el de aboutness. ${ }^{3}$ El comentario, por su parte, comprende la parte

1 Véase por ejemplo los trabajos de Stark (2003), Ewert-Kling (2011) y Dufter y Gabriel (2016). Lo mismo vale para las contribuciones cognitivas, véase por ejemplo Baumann y Schumacher (2012) y Burmester et al. (2014).

2 En lo siguiente los términos enunciado y oración se emplean como sinónimos para referirse a unidades lingüísticas "completas", o sea gramaticales de punto de vista sintáctico, semántico así como entonativo.

3 A pesar de los aspectos problemáticos que se asocian con esa definición del tópico, como sobre todo la incompatibilidad del aboutness-test con ciertos constituyentes, en la gran

Christoph Hülsmann, Universität Salzburg, Salzburg, Austria

¿ Open Access. () 2019 Christoph Hülsmann, published by De Gruyter. (cc) BY-NC-ND This work is licensed under a Creative Commons Attribution-NonCommercial-NoDerivatives 4.0 International License.

https://doi.org/10.1515/9783110605679-006 
complementaria al tópico. Por otro lado, el locutor también puede renunciar a una bipartición (explícita) y realizar un enunciado tético (all-comment). ${ }^{4}$

La tercera dimensión, la estructura foco-trasfondo (FT), también está situada a nivel oracional y concierne a la diferenciación entre los elementos particularmente importantes desde un punto de vista informacional y los elementos menos relevantes. En lo siguiente el término foco se emplea con la definición semántica de Gundel $(1999,295)$ como "the part of the sentence that answers the relevant wh-question (implicit or explicit) in the particular context in which the sentence is used". 5 El trasfondo representa otra vez la categoría complementaria. A diferencia del tópico, que en el caso de enunciados téticos puede estar ausente a nivel segmental, el foco generalmente se analiza como categoría obligatoria y por lo tanto presente en cualquier oración.

Las tres dimensiones informacionales, que se manifiestan simultáneamente en un enunciado, a priori deben ser vistas como independientes, aunque hay preferencias y restricciones en la interacción, tanto en el interior de una lengua como desde una perspectiva interlingüística (cf. Dufter y Jacob 2009, 5). Si bien hay ejemplos como (1), donde se pueden observar correspondencias entre información dada, tópico y trasfondo por una parte (John), y entre información nueva, foco y comentario por otra parte (married Bertha), la oración en (2) muestra que el tópico de un enunciado también puede ser nuevo, y la oración (3) que el foco puede coincidir con información dada.

(1) (Tell me something about John.) -[John] [married Bertha]. (Hinterwimmer 2011, 1876)

(2) [A daughter of a friend of mine], she got her BA in two years. (Gundel 1985, 89)

(3) (So there were two cars, a BMW and a Mercedes. And which car came from the left?) -[The Mercedes] came from the left. (ejemplo modificado de Stutterheim y Klein 2002, 69)

mayoría de las diferentes teorías informacionales ese criterio aún representa "the core of topicality" (Frey 2005, 96).

4 Eso no implica que en enunciados téticos nunca haya ningún elemento topical. De acuerdo con Giurgea y Remberger (2012, 59), también en este tipo de oraciones se pueden asumir tópicos (nulos) implícitos que se refieren al contexto espacio-temporal de los enunciados.

5 Para la definición del foco se recurre a menudo - con referencia al trabajo de Rooth (1985) a la función de señalar y excluir alternativas. En la contribución presente, en cambio, y de acuerdo con Molnár (2006), la contrastividad (tanto explícita como implícita) es vista como categoría independiente, de tipo gradual y capaz de manifestarse no solamente con focos sino también con tópicos. 
Además de las correlaciones que se asumen en la literatura entre las distintas subdimensiones de la estructura informacional, se postulan frecuentemente correspondencias respecto a las otras dimensiones lingüísticas; a saber, categorías sintácticas, semánticas y prosódicas.

Para Molnár (1991, 193), por ejemplo, los tópicos prototípicos se limitan a constituyentes nominales. Según Givón (1976, 152-160) los elementos topicales corresponden más frecuentemente a constituyentes definidos que codifican referentes animados y que desempeñan el papel semántico del agente. Bech y Eide $(2014,3)$ mencionan la tendencia universal del tópico de codificarse como sujeto de la oración. En cuanto a la sucesión lineal de los elementos en la oración, en muchas lenguas, en el caso no marcado, el tópico precede al comentario, y el foco (estrecho) se sitúa en la parte final. A nivel prosódico, la acentuación (secundaria) de tópicos a priori es facultativa, mientras que se asume una relación sistemática entre el foco (estrecho) de la oración y la presencia del acento nuclear. ${ }^{6}$

Aunque hoy en día es poco controvertida la suposición de que la estructura de la información tiene la primacía sobre la sintaxis en el sentido de que puede conducir a modificaciones de patrones sintácticos no marcados (cf. Helfrich y Pöll 2012, 340-341), ${ }^{7}$ los distintos correlatos tienen que ser cuestionados: "[...] such correlations, instead of being an apriori, still have to be established explicitly, partly on empirical grounds" (Dufter y Jacob 2009, 5-6). En ese contexto, la contribución presente ofrece un análisis empírico de los tópicos y los focos lexicales (preverbales) y sus correlatos formales en el español espontáneo, que incluye también el nivel prosódico. Junto a los procedimientos sintácticos, la prosodia, y en especial la configuración tonal de los elementos oracionales, constituye el recurso más relevante para la señalización de categorías informacionales. Pese a la disponibilidad de corpus hablados, aún hoy en día la mayoría de los estudios del habla - como por ejemplo el estudio

\footnotetext{
6 Sin embargo, ya que el acento nuclear señala simultáneamente la fuerza ilocutiva de los enunciados, aquí tampoco se puede postular una correspondencia 1:1.

7 Otro aspecto poco controvertido es la observación de que también la variación diamésica representa un factor relevante en la interfaz de los niveles lingüísticos: "[t]he information flow is different in spoken interaction" (Klein 2012, 105). La naturaleza exacta de las relaciones entre las dimensiones respectivas es, sin embargo, mucho menos evidente. Mientras unos suponen una relación directa entre estructura de la información y sintaxis, otros parten de la idea de una relación indirecta, con la prosodia operando como interfaz. Así, en español a menudo se supone un movimiento del constituyente focal a la posición final, donde puede recibir el acento nuclear y, de esta manera, ser marcado como foco. Apoyándose en el trabajo de Zubizarreta $(1998,124)$ en este contexto generalmente se habla de $p$ (rosodically motivated)movement.
} 
sobre la entonación castellana de Estebas-Vilaplana y Prieto (2010) - se basa en oraciones leídas o discursos semiespontáneos, que frecuentemente provienen de un número limitado de locutores.

\section{El corpus}

El análisis realizado se basa en el corpus C-Oral-Rom de Cresti y Moneglia (2005) que en su totalidad reúne 772 textos que comprenden más de 121 horas y alrededor de 300.000 palabras para cada una de las cuatro lenguas románicas consideradas (español, portugués, francés, italiano). En total participaron 1.427 hablantes en las grabaciones. Debido a la integración del programa WinPitch, que permite visualizar el espectrograma, el oscilograma y la frecuencia fundamental $\left(f_{0}\right)$ junto con las transcripciones ortográficas de los enunciados, el corpus se presta también para análisis prosódicos.

Para el estudio presente se han analizado tres horas y 52 minutos de diálogos espontáneos, producidos por 57 hablantes en contextos informales y grabados en 2001 en Madrid y Segovia. A diferencia de los textos narrativos y monológicos, la conversación dialógica y espontánea se considera la forma básica de comunicación (cf. Kim 1995, 248). Para el análisis se eligieron exclusivamente conversaciones cara a cara. Visto que en la mayoría de los casos el número de los participantes no sobrepasa dos, y dado que las conversaciones tienen lugar en el entorno privado o familiar de los hablantes, se puede asumir un alto grado de dialogicidad y un bajo grado de publicidad (cf. Koch y Oesterreicher 2011, 7). Por regla general, el grado de familiaridad entre los hablantes así como el grado de la experiencia de comunicación común $-\mathrm{y}$ por consecuencia también el common ground (CG, véase Krifka 2007, 15-17) de los locutores- son altos. En cuanto al grado de espontaneidad y de fijación del tema conversacional, se puede constatar cierta variación. En algunas grabaciones los investigadores fijan un tema y guían con preguntas su desarrollo. En la mayoría de los casos, sin embargo, los hablantes deciden espontáneamente sobre el tema y sobre el desarrollo posterior de la comunicación.

\section{Objetivos y metodología}

El objetivo del análisis realizado es doble. Por una parte, se aspira a ilustrar las funciones pragmáticas y discursivas de los constituyentes iniciales a partir de 
datos auténticos; por otra parte, se propone un estudio de los correlatos formales de las categorías funcionales.

Destacándose por su bajo grado de planificación, el estudio del lenguaje espontáneo requiere una metodología particular también a nivel sintáctico. En ciertas construcciones sintácticamente marcadas, el estatus topical de los constituyentes iniciales es poco controvertido. En ese contexto se propone una clasificación de los constituyentes según sus grados de integración sintáctica respecto a la oración principal (cf. Blasco-Dulbecco 1999, 109 y ss.). En el caso de los Chinese style topics, como en (4), la relación entre el constituyente inicial (el ambientillo) y el resto de la oración es de tipo primeramente pragmático. ${ }^{8}$

(4) [TD: visita de B en un estadio de fútbol inglés $]^{9}$

A: Pero hijo, ves el ambientillo del campo, y de los ingleses, de los anormales.

B: ¿Pero qué ambientillo? El ambientillo he ido mil veces a estadios de fútbol, y ya está. (31-82)

Cuando el tópico aparece con la marca preposicional requerida por el verbo, se puede asumir que el locutor ya ha planificado el papel sintáctico del constituyente en el momento del enunciado. Por el alto grado de integración sintáctica -en (5) aparece además de la preposición $a$ un pronombre resasuntivo indicando (de nuevo) la función gramatical del elemento inicial (les) $-^{10}$ las construcciones de ese tipo generalmente se analizan como dislocaciones a la izquierda.

(5) [TD: coches aparcados en la calle] iHombre!, lo que pasa es que luego vino la grúa y a esos dos coches les pondría una multa. (29-22)

En comparación con los tópicos dislocados y los Chinese style topics los tópicos colgados (hanging topics) ocupan una posición intermediaria. En el ejemplo (6) el tópico Flora carece de la marca preposicional requerida por el verbo. $\mathrm{Su}$ función gramatical como complemento indirecto -o como recipiente a nivel de

8 Por la falta de integración sintáctica los Chinese style topics -el término se remonta a la contribución de Chafe $(1976,50)$ - fueron excluidos del análisis.

9 Para asegurar la comprensión de los ejemplos del corpus a pesar del espacio limitado, al principio se indica cada vez el tópico discursivo (TD), o sea el tema de conversación actual (transfrástico) de los interlocutores. Después del ejemplo, el primer número se refiere a la grabación de $C$-Oral-Rom, el segundo a la concreta unidad tonal.

10 Para una sinopsis sincrónica y diacrónica de la duplicación clítica en español, véase Gabriel y Rinke (2010). 
los papeles semánticos- se indica solamente a posteriori con la realización del pronombre clítico se.

(6) [TD: maletas transportadas por avión] Ella me dijo: alguien que fue de Irlanda, en diez días ya tenía lo del avión. Y ella, después de un mes todavía no lo tenía [...] que eso, que Flora todavía no se lo han dado. (29-158)

En otras configuraciones no hay ningún elemento explícito a nivel segmental que señale el grado de integración sintáctica de los constituyentes iniciales en el momento de su realización. Mientras que, en el caso de los complementos directos sin marcación diferencial, como en (7), el orden marcado, así como la presencia de pronombres reasuntivos, permiten una clasificación como topicalizaciones, la situación es menos clara en cuanto a las oraciones que presentan el orden (básico) SVO.

(7) [TD: trabajos de renovación] El parqué más o menos es lo mismo, pero la escayola hay que hacerla. (19-94)

Por el alto grado de lexicalización, no pueden analizarse como tópicos los elementos iniciales en las estructuras la verdad es (que), lo que pasa es (que) y el problema es (que). Los adjetivos nominalizados en oraciones como (8) tampoco corresponden a elementos topicales según el criterio de aboutness, sino que constituyen más bien elementos predicativos.

(8) [TD: novia del padre] Lo gracioso es que ella no sabe que mi padre también anda mal de pelas. (2-194)

En cuanto a los sintagmas nominales "regulares" como en (9), la posición inicial, así como la función de sujeto, pueden considerarse características prototípicas del tópico. ${ }^{11}$ Sin embargo, la comparación con otras lenguas como el francés muestra que una topicalización del sujeto puede realizarse - de manera análoga a las topicalizaciones de objetos- con pronombres reasuntivos como elle en (10). ${ }^{12}$

11 Es preciso recordar que en español la posición preverbal del sujeto se considera no marcada solo en combinación con verbos transitivos y verbos inergativos. Con verbos inacusativos, en cambio, es el orden VS el que se asume como no marcado. Un análisis aparte se impone también para las construcciones con verbos psicológicos como gustar, encantar, importar, molestar, etc. Aquí el orden no marcado corresponde a OVS (cf. Gutiérrez-Bravo 2008, 372ss.).

12 La existencia de estructuras con sujetos topicalizados se explica, en general, con el alto grado de gramaticalización que se observa entre las categorías sujeto y tópico. En francés, por otra parte, también la sucesión de sujeto y pronombre reasuntivo ya se analiza a veces como 
(9) [TD: programas televisivos] Ese programa tuvo un montonazo de audiencia. $(18-91)$

(10) [TD: familia del hablante] Ma grand-mère elle ne savait pas écrire. (5-148) 'Mi abuela no sabía escribir.'

A diferencia de este tipo de lenguas no pro-drop, el español no dispone de pronombres clíticos para marcar de manera explícita la topicalización de sujetos, así que el orden SVO puede corresponder a priori tanto a estructuras tópico-comentario como a oraciones all-focus/all-comment. Ya que en estos casos un análisis sintáctico-pragmático llega a sus límites, es imprescindible incluir también la dimensión prosódica. Según Feldhausen (2014, 120), en italiano también los sujetos iniciales pueden analizarse como topicalizados si presentan -como los tópicos sintácticamente marcados - una frontera prosódica con respecto a los elementos siguientes. Por las semejanzas tipológicas, la observación del autor puede ser aplicada también al español. ${ }^{13}$ Como ilustra la Figura 1, que presenta el espectrograma y la frecuencia fundamental del ejemplo (9), los sujetos topicalizados por regla general se caracterizan por un acento creciente que en el caso de elementos no oxítonos alcanza su máximo en la posición postónica. La presencia sistemática de un tono de frontera alto al final del tópico -en notación autosegmental la configuración topical corresponde a $\mathrm{L}+>\mathrm{H}^{\star} \mathrm{H}-$ está relacionada con las

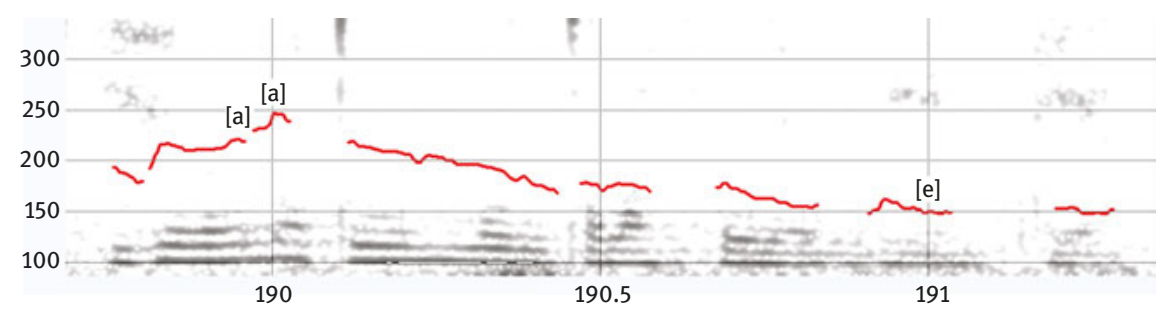

Figura 1: Espectrograma y $\mathrm{f}_{0}$ de Ese programa tuvo un montonazo de audiencia. (18-91).

(semi-)gramaticalizada. Sin embargo, un contraargumento de peso es la imposibilidad de retomar constituyentes indefinidos con pronombres clíticos en oraciones téticas como en (i) (cf. Dufter y Gabriel 2016, 436-437).

(i) ${ }^{\star D}$ Des amis ils sont venus. (Dufter y Gabriel 2016, 437)

'Amigos han llegado.'

13 Eso vale en primer lugar para la partición tópico-comentario. En cuanto a la partición focotrasfondo, sin embargo, la sintaxis del italiano se considera más rígida, ya que excluye los sujetos focales en posición preverbal. 
características tonales del comentario, que típicamente empieza con una entonación más baja y descendente, aumentando así la separación perceptible entre tópico y comentario. Además, la bipartición del enunciado en dos frases intermedias puede (pero no debe de) reforzarse mediante pausas silenciosas y/o sonoras.

En las oraciones con sujetos no marcados, por el contrario, no se puede constatar ningún cambio tonal relevante a comienzos del comentario, y en ciertos casos incluso el sujeto mismo puede carecer de un acento tonal (léxico). Un ejemplo sin frontera entonativa entre sujeto y predicación se da en (11) y en la figura respectiva (Figura 2).

(11) [TD: distribución de vacaciones] Juan Carlos me ha dicho que le envíe eso por fax. (6-341)

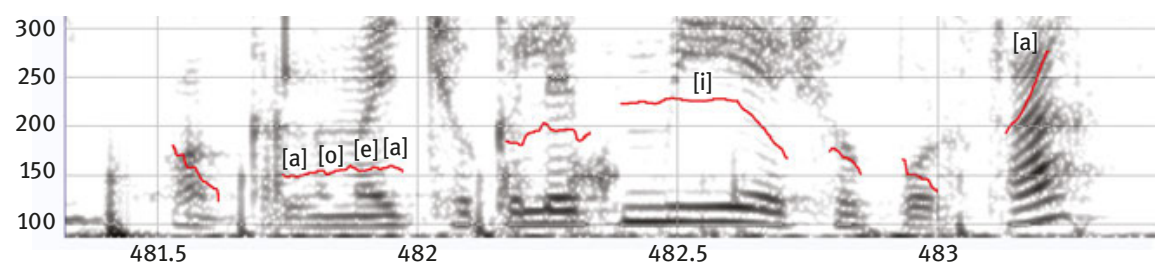

Figura 2: Espectrograma y $\mathrm{f}_{0}$ de Juan Carlos me ha dicho que le envíe eso por fax. (6-341).

\section{Análisis}

Para el análisis se han tomado en cuenta los constituyentes léxicos que aparecen en posición inicial (preverbal) y que forman parte de enunciados (casi) completos. ${ }^{14}$ Como tópicos oracionales (potenciales) sólo se han incluido en el análisis los argumentos sujeto y objeto (directos así como indirectos, o sea preposicionales/dativos). Las oraciones con más de un argumento preverbal se han tomado en cuenta, si al menos uno de ellos aparece en forma léxica. Los adverbios/adjuntos preverbales, que por regla general actúan como frame setting topics indicando las circunstancias espacio-temporales de los enunciados, han quedado desatendidos. Como focos estrechos, en cambio, se han incluido

14 Se han descartado las secuencias sin verbo, cuya frecuencia en el lenguaje espontáneo es relativamente alta. Según Cresti y Moneglia $(2005,14)$ el 36,6\% de los enunciados -en el sentido de actos de habla- del corpus español (entero) carece de verbos (finitos). 
también los constituyentes sintácticamente facultativos (adjuntos/adverbiales así como predicados secundarios).

Para la clasificación básica de los constituyentes se recurrió primeramente -como ilustra la Figura 3- al contexto de los enunciados. Desde un punto de vista informacional los constituyentes analizados desempeñan el papel de tópico, de foco (estrecho) o forman parte de estructuras all-comment/all-focus. ${ }^{15}$

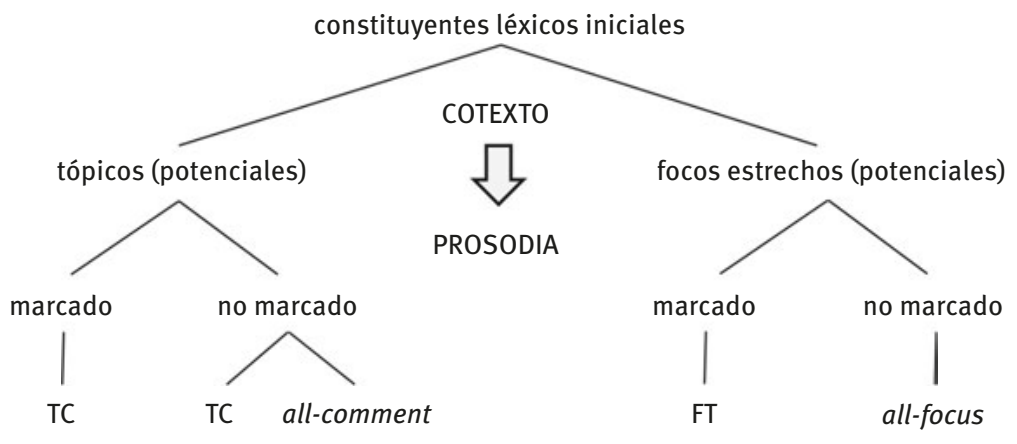

Figura 3: Clasificación informacional de los constituyentes léxicos iniciales.

En un segundo paso también se incluyó la dimensión prosódica. Por la alta correlación que existe entre foco estrecho y acento nuclear, la presencia del último puede ser considerada un factor desambiguante entre configuraciones de foco-trasfondo (FT) y enunciados all-focus. La relación entre tópico (T) y marcación prosódica, en cambio, se presenta como menos sistemática, ya que

15 También han sido excluidos los enunciados interrogativos con constituyentes iniciales que aparecen con partículas interrogativas como en (i). Estos pueden analizarse como focos no marcados. En interrogaciones parciales de tipo (ii), así como en interrogaciones totales como en (iii), en cambio, los constituyentes iniciales sí pueden analizarse como tópicos, sobre los que a continuación el locutor no realiza una predicación, sino que hace una pregunta. Cabe señalar, además, que en la literatura se asume otro tipo de construcción, en la que el constituyente inicial no se analiza ni como tópico ni como foco (contrastivo), sino como verum focus (cf. Leonetti y Escandell-Vidal 2009). El único ejemplo del corpus, con constituyente pronominal, es citado en (iv).

(i) ¿En qué parte de Murcia vive tu madre? (20-170)

(ii) Y el aparato ¿dónde lo habéis cogido? (16-257)

(iii) Y las lámparas ¿ya las ha puesto? (8-230)

(iv) Menos mal que me he librado de algo, porque si no ... De algo me he librado, ¿no? (76-40) 
los tópicos no necesariamente llevan acentos (secundarios) ni presentan una frontera prosódica respecto al comentario (C). ${ }^{16}$

La Tabla 1 indica el número de constituyentes analizados. En cuanto a la duración de las grabaciones, por término medio corresponden a 2,4 tópicos o focos iniciales por minuto. ${ }^{17}$ La sección 4.1. se dedica a enunciados con argumentos iniciales que pueden clasificarse como tópicos. En la sección 4.2. se analizan las oraciones con focos estrechos.

Tabla 1: Número de constituyentes lexicales analizados.

\begin{tabular}{lr}
\hline tópicos (potenciales) & 535 \\
- sujetos & 450 \\
- objetos & 85 \\
focos estrechos & 27 \\
\hline total & 562 \\
\hline
\end{tabular}

\subsection{Tópicos iniciales}

La variación que se puede observar en la planificación del enunciado por parte del locutor constituye una de las propiedades más características del lenguaje hablado: “[...] S[peaker] will often produce a Theme before he has formed a clear idea of what sort of clause he is going to produce about it"18 (Dik 1997, 393). De ahí que, desde un punto de vista funcionalista, los tópicos iniciales normalmente no

16 En la primera parte del capítulo se insistió en la necesidad fundamental de separar en el análisis la articulación tópico-comentario de la estructura foco-trasfondo. No obstante, y de acuerdo con (Molnár 1993, 168), la Figura 3 insinúa que un constituyente no puede funcionar a la vez como base de predicación (tópico) y como elemento predicativo (foco estrecho). Mucho más controvertida es la cuestión de si deben analizarse como téticas las oraciones allfocus (véase por ejemplo Gutiérrez-Bravo 2008, 369) o si son compatibles con una partición tópico-comentario (véase por ejemplo Wehr 2000, 256s.).

17 Aunque para el español el orden SVO se asume como básico, hay que tener en cuenta que en el lenguaje hablado predominan por amplio margen los enunciados sin sujetos léxicos. Tal como se propone desde Du Bois (1987) y han corroborado múltiples estudios en diversas lenguas, los hablantes tienden a realizar solamente un argumento léxico por oración. En oraciones con verbos transitivos, ese argumento léxico aparece normalmente como complemento directo, mientras que en combinación con verbos intransitivos aparece como sujeto. Los sujetos léxicos en oraciones con verbos transitivos son significativamente menos frecuentes.

18 El término tema (theme) se acuñó sobre todo por la Escuela de Praga, mientras que tópico constituye el equivalente anglosajón (cf. Reinhart 1981, 55). 
se analicen como elementos extraídos de la oración principal: "En la tematización, no se extrae el tema de la predicación; al contrario, la predicación se adapta al tema” (Hidalgo Downing 2003, 125). Eso vale sobre todo para los tópicos colgados, como en el ejemplo (12), donde el hablante B retoma el constituyente realizado inmediatamente antes por el interlocutor (José) sin marcar (inicialmente) su función gramatical como complemento indirecto. La realización de la preposición $a$ en el enunciado siguiente puede considerarse un caso de autocorrección.

(12) [TD: planificación de vacaciones]

A: Se lo tendrás que decir a José mañana.

B: Si José ya se lo dije. Yo a José se lo dije el viernes. (6-344)

No obstante, de los 85 tópicos iniciales que no desempeñan el papel sintáctico del sujeto, los tópicos colgados (7 ejemplos) están en minoría. Casi la mitad (41 objetos) concierne a complementos que aparecen con la marca preposicional (adecuada). Esa categoría abarca complementos preposicionales/indirectos (12), argumentos de verbos psicológicos cuya posición inicial es vista como no marcada (13), ${ }^{19}$ así como complementos directos con marcación diferencial (14).

(13) [TD: clases de música] Tenemos niños pequeños, y a los niños no les gusta estudiar, y luego gente mayor que estudia unos días sí y otros no. (1-140)

(14) [TD: novedades sobre conocidos]

A: ¿Y [qué tal] Andrea?

B: Y Andrea, pues muy bien también. No la vi. A Andrea no la vi. (17-210)

Los restantes 37 ejemplos constituyen complementos directos sin marcación diferencial (15). Como muestran los ejemplos (12)-(15), los objetos preverbales por regla general son retomados por pronombres correferenciales. Los casos sin duplicación se explican por la falta de pronombres adecuados, en español, para los sintagmas indefinidos o escuetos (16) y para ciertos objetos preposicionales (17). También en estos contextos, otras lenguas románicas, como el francés, disponen de pronombres respectivos, como demuestran las traducciones en (18)-(19).

19 Gutiérrez-Bravo $(2008,373)$ explica la caracterización del orden OVS como básico en estas estructuras con los papeles semánticos de los complementos. Desde un punto de vista pragmático puede alegarse que, en estos casos, el complemento indirecto experimentante constituye una base de predicación más "probable" que el sujeto, sobre todo cuando se trata de elementos dados, como en (11). Para la correlación entre papeles semánticos y categorías informacionales, véase Brunetti (2011). 
(15) [TD: estudios universitarios de un conocido] Hizo sólo el primer ciclo, ¿no? El segundo ciclo no lo hizo. (21-27)

(16) [TD: versión española de un poema] Si es una traducción al español, tiene que ser una traducción un poco de andar por casa, porque, que yo sepa, traducciones así literarias buenas no Ø hay. (23-85)

(17) [TD: las vacaciones de amigos en La Habana] Y a la playa no Ø fueron, ¿no? (5-64)

(18) De bonnes traductions littéraires comme ça, il n'y en a pas.

(19) Et (à) la plage, ils $\mathbf{y}$ sont pas allés, non?

La gran mayoría de los tópicos iniciales opera como sujeto gramatical de la oración (84\%). Los sintagmas nominales (91\%) -entre los que se constata casi un equilibrio entre referentes animados $(54,9 \%)$ y referentes inanimados $(45,1 \%)$ - predominan sobre los sintagmas preposicionales $(7,7 \%)$ y verbales $(1,3 \%)^{20}$

Se puede observar una fuerte correlación en cuanto a la determinación de los constituyentes iniciales. En el 94,3\% de los casos los elementos son definidos, solo en el 5,7\% se trata de elementos indefinidos. ${ }^{21}$ Frecuentemente, sobre todo en los ejemplos sintácticamente marcados, la presencia de determinantes indefinidos o la falta de determinación no se explican por la primera mención del constituyente en el discurso, sino por la lectura genérica del sintagma topical (20) o por el carácter abstracto del concepto (21).

(20) [TD: reservación de un hueco para aparcar] ¿Pero [.. . ] esa gente había reservado el hueco? ¿No tienen que pagar al ayuntamiento o algo por el estilo? [...] O llegas, colocas la señal y ya está. [...] Claro, si la gente se conoce de cómo va el tema, ellos, jah!, una señal, la quitas y punto. (29-19)

20 Además de infinitivos como en (i) se han documentado subordinadas topicales en oraciones complejas, como la proposición sustantiva en (ii).

(i) [TD: estudiar] Había un montón de tíos míos que se habían quedado en Madrid. Y venían a comer a casa, venían a cenar, no sé qué, y ponerte a estudiar era imposible. (8-194)

(ii) [TD: programas televisivos] En un medio privado pueden poner lo que les dé la gana. Pero que en un medio público pongan eso, a mí no me parece ético. (18-116)

21 Pese a la ausencia de determinantes, los nombres propios pueden considerarse elementos inherentemente definidos. 
(21) [TD: educación de niños] Hay mucho problema con eso. Yo, los míos son grandecitos, pero educación no tienen ninguna. (15-49)

Ya que no existe una correspondencia 1:1 entre la presencia/ausencia de determinantes (in)definidos y el carácter dado o nuevo de los constituyentes, la última dimensión necesita de un análisis aparte. El 67,1\% de los constituyentes iniciales representa referentes dados en el discurso. De acuerdo con Baumann y Riester (2012), en este contexto hace falta diferenciar dos tipos de givenness, el primero situado a nivel léxico, el segundo a nivel referencial. La necesidad de diferenciación se manifiesta con ejemplos de tipo de (22), donde el sintagma preposicional a tu compañera es correferencial con Esther y por lo tanto referencialmente dado al momento de la enunciación, pero nuevo a nivel léxico.

(22) [TD: clientes molestos] Siguieron allí, yo superborde. El otro que se quería llevar a Esther: “No, no, y a tu compañera ¿no me la vendes?” (7-181)

Según el grado de activación de los elementos dados, se pueden diferenciar como funciones discursivas la reintroducción de un elemento como tópico oracional como en (20)-(21) y la continuación inmediata de un tópico activado como en (13) y (22). En el caso de que la mención previa sea realizada por el interlocutor, como en los ejemplos (12) y (14), la continuación equivale a la aceptación de un constituyente como tópico oracional por parte del locutor. ${ }^{22}$

En el 5,4\% de los casos el constituyente inicial combina elementos dados con elementos nuevos, como en (15), donde el locutor repite el lexema nominal (ciclo) cambiando sólo el adjetivo ordinal. Un cuarto de los tópicos $(25,1 \%)$ corresponde a referentes nuevos, o sea no mencionados de manera explícita en el discurso anterior. ${ }^{23}$ A nivel discursivo, se trata de la introducción de un nuevo tópico y, con ello, del cambio del tópico de la oración previa. Según el modelo de Prince (1981), los referentes no dados pueden diferenciarse en referentes accesibles por el contexto extralingüístico o a partir de conceptos dados (inferrable), como referentes disponibles a nivel del saber general o específico del hablante (unused), o como referentes no disponibles (brand-new).

El corpus analizado contiene exclusivamente ejemplos de las dos primeras categorías. La accesibilidad del tópico semántica en (23), por ejemplo, se puede explicar por la activación previa del concepto hiperonímico asignaturas así

22 Como tal puede convertirse a continuación en un tópico discursivo.

23 En los restantes casos (2,4\%) la ausencia del cotexto en el corpus no permite una clasificación válida. 
como por el saber del interlocutor sobre los estudios (lingüísticos) del hablante. En (24), que presenta una combinación de dos constituyentes léxicos preverbales, Vidal es nuevo en el discurso (a diferencia de potaje), pero el referente tiene - ya que se trata de un miembro de la familia - una representación mental en la cognición de los interlocutores (véase en ese contexto también el enfoque semántico del file-card-model de Reinhart 1981 y Heim 1988, así como el modelo de Vallduví 1992).

(23) [TD: estrés universitario] Este cuatrimestre es como si tuviera diez asignaturas. [.. I] Y encima es eso, de semántica es que estoy súper perdida, porque no tengo ni idea de nada. (13-85)

(24) [TD: preferencias culinarias] Porque tengo yo mi arreglo, mi comida. Hoy yo sé que el potaje a Vidal le gusta. (10-46)

La única oración cuyo argumento inicial a primera vista podría caracterizarse como brand-new es citada en (25).

(25) [TD: deseo de un perro]

A: Porque Nesca ha tenido camada, y ha tenido diez perros, y yo llevaba un mes pidiéndoles un bóxer atigrado, porque Pilar, la señora que cose para mi madre, la señora esta mayor,

B: Sí.

A: pues le encantan los bóxer atigrados. (7-4)

Después de pronunciar el nombre propio Pilar, la hablante A se da cuenta de que el interlocutor posiblemente no está en grado de identificar el referente, por lo cual continúa su enunciado con aposiciones explicativas. Solo después de que el interlocutor señale el éxito de descodificación mediante la partícula afirmativa sí (lo que presupone la existencia de una "ficha mental" del referente en la cognición de $\mathrm{B})$, sigue con la parte predicativa de la oración.

A nivel prosódico, el análisis del corpus ha podido mostrar que, independientemente de las funciones gramaticales, la mayoría absoluta de los constituyentes iniciales $(86,7 \%)$ lleva un acento tonal (secundario), considerado requisito previo para la suposición de frases intermedias (cf. Féry 2010, 273). Frecuentemente, en los $42,4 \%$ de las oraciones del corpus entero, además se puede constatar una frontera entonativa respecto de los elementos siguientes. Mientras que en los casos ya sintácticamente marcados esta configuración tonal puede ser vista como procedimiento de señalación adicional, para los sujetos iniciales - como para el proparoxítono árbitro en (26) y la figura respectiva 
(Figura 4) - constituye el factor primordial permitiendo un análisis de los sujetos preverbales como topicalizados.

(26) [TD: baloncesto] Y el árbitro pues pita fuera de juego. (3-35)

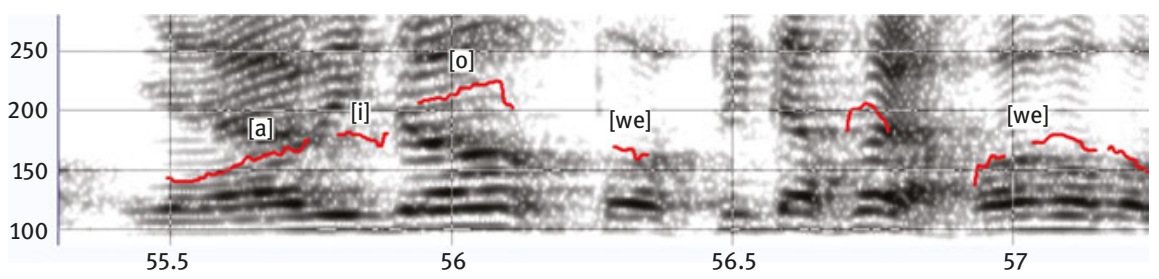

Figura 4: Espectrograma y $\mathrm{f}_{0}$ de $Y$ el árbitro pues pita fuera juego. (3-35).

\subsection{Focos iniciales}

A diferencia del tópico, el foco también puede constituirse de elementos no referenciales. Además, pueden ser focales constituyentes sintácticamente facultativos (adjuntos/adverbiales). Como focos obtienen, desde un punto de vista pragmático, un estatus comparable a argumentos a nivel sintáctico, ya que su realización -como ilustra el ejemplo (27) - se hace obligatoria para el hablante cooperativo (cf. Goldberg y Ackerman 2001, 798-799). ${ }^{24}$

\section{(27) [TD: planes para la Semana Santa]}

A: Pero tú, si te vas, ¿cuándo te vas?

B: Hombre, yo, si os fuerais vosotros, nos íbamos el, el do-, JUEves. (22-65)

Mientras que la posición preverbal del tópico corresponde al orden informacional no marcado, el foco estrecho en español normalmente se sitúa -como en el ejemplo (27) - en la posición final de la oración (declarativa). Los enfoques sintáctico-formales generalmente insisten en que la anteposición no es compatible con los focos informacionales, sino sólo se admite en casos de focos contrastivos (cf. Molnár 2006, 211-212). En esos estudios la noción de contraste se limita frecuentemente al foco correctivo, que presupone la mención previa de un constituyente de la misma clase denotativa, como en (28), donde el atributo preposicional de deportes contrasta con el co-hipónimo de debate (para los diferentes tipos de focos contrastivos, véase el trabajo de Dik et al. 1981). En (29) es

24 En los ejemplos siguientes la presencia del acento nuclear se señala con mayúsculas. 
citado otro ejemplo de un foco correctivo, que muestra además que el foco oracional (Nuria) puede corresponder a información dada en el discurso anterior.

(28) [TD: programas televisivos]

A: Bueno, vale, pues ahora van a poner en lugar de Tómbola un programa de debate.

B: Pero de dePORtes han dicho que lo iban a hacer. (18-97)

(29) [TD: películas]

A: ¿Y Nuria lo sabe? [. . _] ¿Tú fuiste la que me dijiste que "El Bola" era una porquería?

B: No. NUria dijo que "El Bola" era una porquería. (4-147)

Sin embargo, el ejemplo (30), donde el constituyente focal responde a una pregunta explícitamente realizada, muestra la admisibilidad de focos informacionales.

(30) [TD: acontecimiento pasado]

A: ¿Y cuándo lo h[izo]?

B: ¡Ah!, pues el año paSAdo fue. (12-158)

Además, el ejemplo (31), en el que el hablante realiza una autocorrección, subraya la inadecuación de una clasificación dicotómica en foco informacional y foco contrastivo, ya que presenta una combinación de los dos tipos de focos. En ese ejemplo, el martes por un lado responde a la pregunta realizada (por el hablante mismo); por otro lado, contrasta con la alternativa el miércoles.

(31) [TD: paradero del hablante] ¿Cuándo estuve en casa? el miércoles o por ahí, o el MARtes estuve, que estuvisteis alli en el pueblo, ¿no? (9-122)

En otros casos la posición inicial del foco se explica con la improbabilidad o la imprevisibilidad de la información codificada por el constituyente. Un ejemplo de esos focos mirativos es citado en (32). Aquí el sintagma preposicional en dos días, enfatizado por la repetición en forma reducida en posición posverbal (en dos), corresponde a un lapso de tiempo considerado como insólito para la fabricación de chalecos.

(32) [TD: chaleco]

A: A ver, el chaleco. Dice la Inma que le hagas uno a ella. Yo no quiero. [...] y qué, te lo haces en seguida?

B: ¡Anda!, en dos DÎ́as me lo he hecho, en dos. (12-59) 
Otro foco mirativo comparable es el sintagma preposicional en diez días en (33). Por la realización tanto anterior como posterior de un mes, el foco a la vez puede analizarse como contrastivo. El espectrograma correspondiente (Figura 5) muestra la entonación típica de oraciones con focos iniciales. En la sílaba tónica se constata un acento creciente al que sigue un descenso en la sílaba postónica. Los elementos posfocales se caracterizan por una entonación baja y plana (en notación autosegmental corresponde a la configuración $\mathrm{L}+\mathrm{H}^{\star} \mathrm{L}$ ).

(33) [TD: mudanza]

A: Flora me ha dicho que se fue de la casa provisional a la definitiva después de un mes y todavía no le había llegado nada, o sea las cosas que iban por avión.

B: Sí, van lentas.

A: Imagínate, no, no, que de, ella me dijo, alguien que fue de Irlanda, en diez días ya tenía lo del avión, y ella, después de un mes todavía no lo tenía. (29-154)

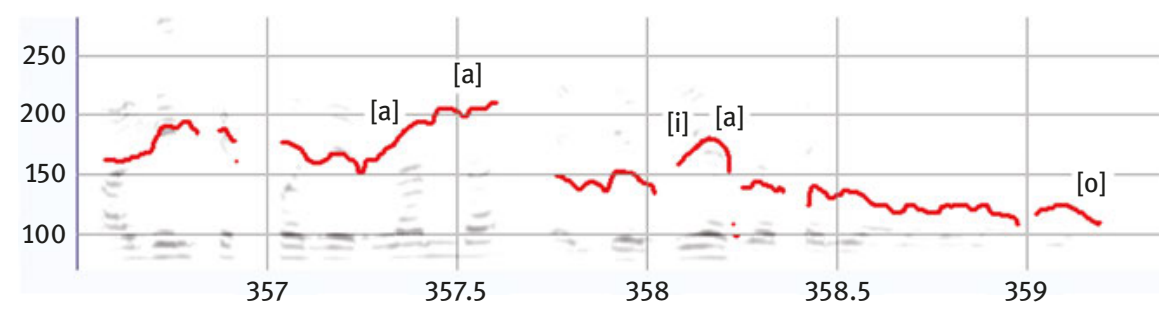

Figura 5: Espectrograma y $\mathrm{f}_{0}$ de Alguien que fue de Irlanda, en diez días ya tenía lo del avión. (29-154).

En la literatura, el carácter enfático o expresivo de la estructura se menciona frecuentemente como otro factor relevante de la anteposición del foco. ${ }^{25}$ Sin embargo, la distribución de los focos iniciales no se restringe ni a constituyentes contrastivos ni a información improbable o enfática. En el ejemplo (34) el constituyente focal no codifica información imprevisible sino todo lo contrario, es decir información presupuesta, como indica la presencia del question tag (¿no?).

25 En ese contexto Abeillé et al. (2009) proponen para la estructura análoga en francés la denominación dramatic extraction. 
(34) [TD: conocido de los hablantes]

A: Ah, pues eso, que me llamó ayer por teléfono, y me dejó un mensaje [.. .] supongo que habia estado con los chavales en Francia aunque, lo que, lo que nos contó, que tenía que marchar y eso.

B: Sí, en un viaje de eSTUdios era, ¿no? (13-6)

En total, se documentaron en el corpus 27 ejemplos de focos estrechos iniciales. Además de frases nominales, que constituyen otra vez la mayoría (15 ejemplos), se hallan frases preposicionales (6 ejemplos) y adjetivales (6 ejemplos). A nivel gramatical, se trata de adverbiales (9 ejemplos), predicados secundarios de sujeto $\mathrm{u}$ objeto (8 ejemplos), complementos directos (5 ejemplos), sujetos (4 ejemplos) y el atributo al complemento directo citado más arriba en (28).

A los 19 ejemplos de oraciones declarativas se añaden 8 casos de focos exclamativos, que pueden analizarse como subtipos enfáticos en los que la sucesión foco-trasfondo corresponde al orden no marcado. En esos enunciados exclamativos, en tres ejemplos los focos siguen a la partícula qué como elementos nominales (35) y en cinco ejemplos como elementos adjetivales (36). Entre los adjetivos focales, que pueden servir para expresar ironía, como en (36) (para la relación entre anteposición de focos e ironía, véase la contribución de Escandell-Vidal 2014), aparecen exclusivamente elementos graduales/evaluativos. Además de simpático se trata de los adjetivos malo, guapo, mono y soso.

(35) [TD: recuerdos de A]

A: El mayor susto de mi vida, cuando, cuando la, la caldera [...] y salió el ratón [...].

B: ¡Qué SAltos pegabas! (6-43)

(36) [TD: estado de ánimo del interlocutor] ¡Qué simPÁtica eres! No me gusta cómo sonríes. Estás sonriendo, pero estás enfadada conmigo. (26-153)

En (otras) tres oraciones exclamativas, como por ejemplo en (37), el constituyente subsiguiente a qué no lleva el acento nuclear, sino solamente un acento secundario, por lo cual puede analizarse como parte de un enunciado all-focus. En general, las estructuras exclamativas necesitan de un análisis aparte y más detallado, ya que presentan a menudo propiedades prosódicas particulares.

(37) ¡Jo!, ¡qué SUEño me está enTRANdo! (14-183) 


\section{Conclusión}

Los resultados del estudio de corpus generalmente confirman la poca frecuencia de argumentos (sujeto y objeto) léxicos preverbales en el lenguaje hablado. Entre los argumentos que se realizan en forma léxica, el análisis ha mostrado que a la mayoría se le puede atribuir una función topical. Desde un punto de vista pragmático así como cognitivo, la articulación tópico-comentario representa el orden informacional no marcado. Independientemente de las funciones gramaticales que desempeñan los tópicos - en la gran mayoría de los casos operan como sujetos de la oración- constituyen ya sea elementos mencionados de forma explícita en el discurso anterior, o accesibles por el co(n)texto. Otra correlación esperada concierne al carácter definido de los tópicos iniciales. Se ha subrayado, además, la necesidad de incluir en los análisis informacionales también el nivel prosódico, sobre todo en relación con los sujetos lexicales, donde las configuraciones entonativas permiten la diferenciación entre los constituyentes no marcados y los sujetos topicalizados.

Las oraciones con focos estrechos en posición preverbal, por el contrario, corresponden a la configuración informacional marcada. Pese a los ejemplos menos numerosos, se ha podido documentar que la contrastividad no basta para explicar la distribución de focos iniciales. De acuerdo con Downing (1995, 20) se puede llegar a la conclusión de que la variación sintáctica que se observa en el lenguaje hablado no debería ser vista como una desviación de un estándar, sino más bien como ilustración de las posibilidades sintácticas de una lengua. Desde una perspectiva primariamente funcional, de hecho, la suposición de un orden sintácticamente determinado puede relativizarse, en la medida en que en toda situación de comunicación natural -también al inicio de conversaciones - existen un contexto extralingüístico y un common ground que determinan en alto grado la forma concreta de los enunciados.

\section{Agradecimientos}

Quiero dar las gracias a los dictaminadores externos por sus comentarios, así como a Mireia Esteban Martínez por su trabajo de revisión.

\section{Referencias}

Abeillé, Anne, Danièle Godard y Frédéric Sabio. 2009. The Dramatic Extraction Construction in French. Bucharest Working Papers in Linguistics 11(1). 135-148. 
Baumann, Stefan y Arndt Riester. 2012. Referential and Lexical Givenness. Semantic, Prosodic and Cognitive Aspects. En Gorka Elordieta (ed.). Prosody and Meaning, 119-162. Berlin: De Gruyter.

Baumann, Stefan y Petra Schumacher. 2012. (De-)Accentuation and the Processing of Information Status. Evidence from Event-Related Brain Potentials. Language and Speech 55(3). 361-381.

Bech, Kristin y Kristine Gunn Eide. 2014. Information Structure and Syntax in Old Germanic and Romance Languages. En Kristin Bech y Kristine Gunn Eide (eds.). Information Structure and Syntactic Change in Germanic and Romance Languages, 1-14. Amsterdam/ Philadelphia: Benjamins.

Blasco-Dulbecco, Mylène. 1999. Les dislocations en français contemporain. Étude syntaxique. Paris: Honoré Champion.

Brunetti, Lisa. 2011. Interaction entre topique, structure thématique et discours en italien et en espagnol. En Andreas Dufter y Daniel Jacob (eds.). Syntaxe, structure informationnelle et organisation du discours dans les langues romanes, 9-27. Francfort-sur-le-Main: Lang.

Burmester, Juliane, Katharina Spalek e Isabell Wartenburger. 2014. Context Updating during Sentence Comprehension. The Effect of Aboutness Topic. Brain \& Language 137. 62-76.

Chafe, Wallace. 1976. Givenness, Contrastiveness, Definiteness, Subjects, Topics, and Point of View. En Charles Li, (ed.). Subject and Topic, 25-55. New York: Academic.

Cresti, Emanuela y Massimo Moneglia (eds.). 2005. C-ORAL-ROM. Integrated Reference Corpora for Spoken Romance Languages. Amsterdam/Philadelphia: Benjamins.

Dik, Simon. 1997. The Theory of Functional Grammar, vol. 2. Berlin/New York: De Gruyter.

Dik, Simon, Maria Hoffman, Jan de Jong, Sie Ing Djang, Harry Stroomer y Lourens de Vries. 1981. On the Typology of Focus Phenomena. En Teun Hoekstra (ed.). Perspectives on Functional Grammar, 41-74. Dordrecht: Foris.

Downing, Pamela. 1995. Word Order in Discourse. By Way of Introduction. En Pamela Downing y Michael Noonan (eds.). Word Order in Discourse, 1-27. Amsterdam/Philadelphia: Benjamins.

Du Bois, John (1987). The Discourse Basis of Ergativity. Language 63. 805-855.

Dufter, Andreas y Christoph Gabriel. 2016. Information Structure, Prosody and Word Order. En Susann Fischer y Christoph Gabriel (eds.). Manual of Grammatical Interfaces in Romance, 419-455. Berlin: De Gruyter.

Dufter, Andreas y Daniel Jacob. 2009. Introduction. En Andreas Dufter y Daniel Jacob (eds.). Focus and Background in Romance Languages, 1-18. Amsterdam/Philadelphia: Benjamins.

Escandell-Vidal, Victoria y Manuel Leonetti. 2014. Fronting and Irony in Spanish. En Andreas Dufter y Álvaro Octavio de Toledo (eds.). Left Sentence Peripheries in Spanish. Diachronic, Variationist and Comparative Perspectives, 309-342. Amsterdam/ Philadelphia: Benjamins.

Estebas-Vilaplana, Eva y Pilar Prieto. 2010. Castilian Spanish Intonation. En Pilar Prieto y Paolo Roseano (eds.). Transcription of Intonation of the Spanish Language, 17-49. München: LINCOM.

Ewert-Kling, Karin. 2011. Le modèle des trois dimensions informatives. En Andreas Dufter y Daniel Jacob (eds.). Syntaxe, structure informationnelle et organisation du discours dans les langues romanes, 79-93. Francfort-sur-le-Main: Lang.

Feldhausen, Ingo. 2014. Intonation and Preverbal Subjects in Italian. En Stefan Fuchs, Martine Grice, Anne Hermes, Leonardo Lancia y Doris Mücke (eds.). Proceedings of the 10th 
International Seminar on Speech Production, Köln, 5-8 May 2014, 118-121, Cologne: University of Cologne.

Féry, Caroline. 2010. Syntax, Information Structure, Embedded Prosodic Phrasing, and the Relational Scaling of Pitch Accents. En Nomi Erteschik-Shir y Lisa Rochman (eds.). The Sound Patterns of Syntax, 271-290. Oxford/New York: Oxford University Press.

Frey, Werner. 2005. Pragmatic Properties of Certain German and English Left Peripheral Constructions. Linguistics 43(1). 89-129.

Gabriel, Christoph y Esther Rinke. 2010. Information Packaging and the Rise of Clitic Doubling in the History of Spanish. En Gisella Ferraresi y Rosemarie Lühr (eds.). Diachronic Studies on Information Structure. Language Acquisition and Change, 63-86. Berlin/New York: De Gruyter.

Giurgea, Ion y Eva-Maria Remberger. 2012. Zur informationsstrukturellen Konfiguration und Variation postverbaler Subjekte in den romanischen Null-Subjekt-Sprachen. Zeitschrift für Sprachwissenschaft 31(1). 43-99.

Givón, Talmy. 1976. Topic, Pronoun and Grammatical Agreement. En Charles Li (ed.). Subject and Topic, 149-188. New York: Academic.

Goldberg, Adele y Farrell Ackerman. 2001. The Pragmatics of Obligatory Adjuncts. Language 77(4). 798-814.

Gundel, Jeanette. 1985. 'Shared Knowledge’ and Topicality. Journal of Pragmatics 9(1). 83-107.

Gundel, Jeanette. 1999. On Different Kinds of Focus. En Peter Bosch y Rob van der Sandt, (eds.). Focus. Linguistic, Cognitive, and Computational Perspectives, 293-305. Cambridge: University Press.

Gutiérrez-Bravo, Rodrigo. 2008. La identificación de los tópicos y los focos. Nueva Revista de Filología Hispánica 56. 363-401.

Heim, Irene. 1988. The Semantics of Definite and Indefinite Phrases. New York/London: Garland.

Helfrich, Uta y Bernhard Pöll. 2012. Wortstellung und Informationsstruktur. En Joachim Born, Bernhard Pöll, Christopher Laferl y Robert Folger (eds.). Handbuch Spanisch. Sprache, Literatur, Kultur, Geschichte in Spanien und Hispanoamerika. Für Studium, Lehre, Praxis, 340-345. Berlin: Schmidt.

Hidalgo Downing, Raquel. 2003. La tematización en el español hablado. Estudio discursivo sobre el español peninsular. Madrid: Gredos.

Hinterwimmer, Stefan. 2011. Information Structure and Truth-Conditional Semantics. En Klaus von Heusinger, Claudia Maienborn y Paul Portner (eds.). Semantics. An International Handbook of Natural Language Meaning, 1875-1908. Berlin: De Gruyter.

Hockett, Charles. 1958. A Course in Modern Linguistics, New York: Macmillan.

Kim, Kyu-hyun. 1995. Wh-Clefts and Left-Dislocation in English Conversation. En Pamela Downing y Michael Noonan (eds.). Word Order in Discourse, 247-296. Amsterdam/ Philadelphia: Benjamins.

Klein, Wolfgang. 2012. The Information Structure of French. En Manfred Krifka y Renate Musan (eds.). The Expression of Information Structure, 95-126. Berlin: De Gruyter.

Koch, Peter y Wulf Oesterreicher. 2011. Gesprochene Sprache in der Romania, 2nd edition. Berlin: De Gruyter.

Krifka, Manfred. 2007. Basic Notions of Information Structure. Interdisciplinary Studies on Information Structure 6. 13-55. 
Leonetti, Manuel y Victoria Escandell-Vidal. 2009. Fronting and Verum Focus in Spanish. En Andreas Dufter y Daniel Jacob (eds.). Focus and Background in Romance Languages, 155-204. Amsterdam/Philadelphia: Benjamins.

Molnár, Valéria. 1991. Das Topik im Deutschen und im Ungarischen. Stockholm: Almqvist \& Wiksell.

Molnár, Valéria. 1993. Zur Pragmatik und Grammatik des TOPIK-Begriffes. En Marga Reis (ed.). Wortstellung und Informationsstruktur, 155-202. Tübingen: Niemeyer.

Molnár, Valéria. 2006. On Different Kinds of Contrast. En Valéria Molnár y Susanne Winkler (eds.). The Architecture of Focus, 197-233. Berlin: De Gruyter.

Prince, Ellen. 1981. Toward a Taxonomy of Given-New Information. En Peter Cole (ed.). Radical Pragmatics, 223-255. New York: Academic Press.

Prince, Ellen. 1992. The ZPG Letter. Subjects, Definiteness, and Information-Status. En William Mann y Sandra Thompson (eds.). Discourse Description. Diverse Linguistic Analyses of a Fund-Raising Text, 295-325. Amsterdam: Benjamins.

Reinhart, Tanya. 1981. Pragmatics and Linguistics. An Analysis of Sentence Topics. Philosophica 27(1). 53-94.

Rooth, Mats. 1985. Association with Focus. Tesis de Doctorado. University of Massachusetts.

Stark, Elisabeth. 2003. Wortstellung und Informationsstruktur. En Ingo Kolboom, Thomas Kotschi y Edward Reichel (eds.). Handbuch Französisch. Sprache, Literatur, Kultur, Gesellschaft. Für Studium, Lehre, Praxis, 301-309. Berlin: Schmidt.

Stutterheim, Christiane von y Wolfgang Klein. 2002. Quaestio and L-Perspectivation. En Carl Graumann y Werner Kallmeyer (eds.). Perspective and Perspectivation in Discourse, 59-88. Amsterdam: Benjamins.

Vallduví, Enric. 1992. The Informational Component. Tesis de Doctorado. University of Pennsylvania.

Wehr, Barbara. 2000. Zur Beschreibung der Syntax des français parlé (mit einem Exkurs zu 'thetisch' und 'kategorisch'). En Barbara Wehr y Helga Thomaßen (eds.). Diskursanalyse. Untersuchungen zum gesprochenen Französisch. Akten der gleichnamigen Sektion des 1. Kongresses des Franko-Romanisten-Verbands (Mainz, 23. - 26. September 1998), 239-289. Frankfurt a.M.: Lang.

Zubizarreta, María. 1998. Prosody, Focus, and Word Order. Cambridge/London: MIT Press. 